\title{
THERMOMECHANICAL CONSTRAINTS AND CONSTITUTIVE FORMULATIONS IN THERMOELASTICITY
}

\author{
S. BAEK AND A. R. SRINIVASA
}

Received 7 December 2002

We investigate three classes of constraints in a thermoelastic body: (i) a deformationtemperature constraint, (ii) a deformation-entropy constraint, and (iii) a deformationenergy constraint. These constraints are obtained as limits of unconstrained thermoelastic materials and we show that constraints (ii) and (iii) are equivalent. By using a limiting procedure, we show that for the constraint (i), the entropy plays the role of a Lagrange multiplier while for (ii) and (iii), the absolute temperature plays the role of Lagrange multiplier. We further demonstrate that the governing equations for materials subject to constraint (i) are identical to those of an unconstrained material whose internal energy is an affine function of the entropy, while those for materials subject to constraints (ii) and (iii) are identical to those of an unstrained material whose Helmholtz potential is affine in the absolute temperature. Finally, we model the thermoelastic response of a peroxidecured vulcanizate of natural rubber and show that imposing the constraint in which the volume change depends only on the internal energy leads to very good predictions (compared to experimental results) of the stress and temperature response under isothermal and isentropic conditions.

\section{Introduction}

A purely mechanical constraint such as incompressibility or inextensibility is characterized by a restriction on the class of possible motions. It is typically assumed that the stress arising from the constraints is workless (Truesdell and Noll [13, page 70]). Green et al. [5] considered a thermoelastic constraint in the form of a function linking the deformation and temperature and showed that, in general, the energy equation is also affected by such constraints. They assumed that the constraint stress is workless for isothermal processes. However, Chadwick and Scott [4] found that the deformation-temperature constraints result in instabilities in wave propagation in a thermoelastic solid and Scott suggested in [10] that the constraints linking the entropy and deformation result in stable solutions. Scott, in his subsequent work $[11,12]$, investigated the deformation-temperature and deformation-entropy constraints as limits of unconstrained thermoelastic solids. He 
showed that the linear form of a constraint connecting the deformation gradient and temperature results in a negative heat capacity which corresponds to a loss of stability. Scott [12] recognized that the limit of the specific heat at constant deformation being zero is equivalent to the limit of a deformation-entropy constraint.

In this paper, we investigate thermoelastic constraints as limits of unconstrained materials, using the procedure outlined by the authors in [1]. In order to do this, we introduce three different but equivalent formulations of thermoelasticity; namely, the $\{\mathbf{F}, \theta\}$ formulation, the $\{\mathbf{F}, \eta\}$ formulation, and the $\{\mathbf{F}, \varepsilon\}$ formulation. Three cases of constraints are considered: (i) $\theta=\hat{\theta}(\mathbf{F})$, (ii) $\eta=\hat{\eta}(\mathbf{F})$, and (iii) $\varepsilon=\hat{\varepsilon}(\mathbf{F})$.

The principal results in this paper are the following ones.

(1) The constraint $\theta=\hat{\theta}(\mathbf{F})$ leads to either the specific heat or the bulk modulus tending to $-\infty$ which implies unstable behavior-a well known result — see Scott $[10,12]$, and Knops and Wilkes [8].

(2) The constraints $\eta=\hat{\eta}(\mathbf{F})$ and $\varepsilon=\hat{\varepsilon}(\mathbf{F})$ are equivalent.

(3) In the above cases, the temperature plays the role of the Lagrange multiplier.

(4) The constitutive equations for materials subject to the constraint $\theta=\hat{\theta}(\mathbf{F})$ are the same as those for an unconstrained material whose internal energy is given by

$$
\varepsilon=\hat{\psi}(\mathbf{F})+\eta \hat{\theta}(\mathbf{F}) .
$$

(5) The constitutive equations for materials subject to the constraints $\eta=\hat{\eta}(\mathbf{F})$ or $\varepsilon=$ $\hat{\mathcal{E}}(\mathbf{F})$ are the same as those for an unconstrained material whose Helmholtz potential is given by

$$
\psi=\hat{\varepsilon}(\mathbf{F})-\theta \hat{\eta}(\mathbf{F})
$$

(6) The assumption $\varepsilon=\hat{\varepsilon}(J)$ leads to good agreement with experimental data for peroxide-cured vulcanizate of rubber (see Chadwick [3]).

In order to help in understanding the behavior of the material and in interpreting the constraint response, we follow [1] and consider constraints as limits of unconstrained materials. This procedure also avoids certain difficulties in specifying the properties of constraint responses (such as worklessness, etc.). This limiting process is similar to that suggested by Scott [12] but the derived quantities are obtained at the limit without augmenting the various potentials as done by Scott [12].

In Section 4, we choose a specific form for the Helmholtz free energy, which is equivalent to the imposition of a deformation-energy constraint, study the response of the material for homogeneous deformations, and compare the stress and temperature response with unconstrained cases and experimental data.

\section{Thermomechanical formulation for unconstrained thermoelasticity}

We consider a thermoelastic solid $\mathscr{B}$ occupying a reference configuration $\kappa_{0}(\mathscr{B})$ at time $t=0$ and a configuration $\kappa_{t}(\mathscr{B})$ at time $t$. Let the position of a material particle be $\mathbf{X}$ and $\mathbf{x}$ in these two configuration, respectively. The motion measured from a reference 
configuration $\kappa_{0}$ and deformation gradient are given, respectively, as

$$
\mathbf{x}=\chi_{\kappa_{0}}(\mathbf{X}, t), \quad \mathbf{F}=\frac{\partial \chi_{\kappa_{0}}}{\partial \mathbf{X}},
$$

with determinant $J=\operatorname{det} \mathbf{F}$. The densities $\rho_{0}$ in $\kappa_{0}(\mathscr{B})$ and $\rho$ in $\kappa_{t}(\mathscr{B})$ are related by

$$
\rho_{0}=J \rho,
$$

expressing the conservation of mass.

In order to elucidate the precise nature of various constraints in thermoelasticity, we will begin with various equivalent formulations of the constitutive equations for such variables. These formulations differ in the set of independent variables used in the constitutive theory.

Our first formulation will be based on the familiar Helmholtz potential. Here, the independent variables are the deformation gradient $\mathbf{F}$ and the temperature $\theta$. The equation of state, from which the stress $\mathbf{T}$ response and the entropy $\eta$ are obtained, is given as

$$
\psi=\hat{\psi}(\mathbf{F}, \theta)
$$

where $\psi$ is the specific Helmholtz potential. The Cauchy stress $\mathbf{T}$ and the entropy per unit mass $\eta$ are obtained from $\psi$ as

$$
\begin{aligned}
& \mathbf{T}=\rho \frac{\partial \hat{\psi}}{\partial \mathbf{F}} \mathbf{F}^{T}, \\
& \eta=-\frac{\partial \hat{\psi}}{\partial \theta}
\end{aligned}
$$

where $\eta$ is assumed to be a monotonically increasing function with respect to $\theta$. We will refer to (2.3), (2.4), and (2.5) as the Helmholtz potential form of the equations of state or simply the $\{\mathbf{F}, \theta\}$ formulation. The constitutive expression can be rewritten in the energetic form $(\{\mathbf{F}, \eta\}$ formulation) using the Legendre transformation

$$
\varepsilon=\psi-\theta \frac{\partial \psi}{\partial \theta}
$$

Solving (2.5) for $\theta$ in terms of $\eta$ and $\mathbf{F}$ and substituting the result into (2.6), we obtain

$$
\varepsilon=\hat{\varepsilon}(\mathbf{F}, \eta)
$$

Now using (2.7) as the equation of state with $\mathbf{F}$ and $\eta$ as independent state variables, the stress and the absolute temperature are obtained as

$$
\begin{aligned}
& \mathbf{T}=\rho \frac{\partial \hat{\varepsilon}}{\partial \mathbf{F}}, \\
& \theta=\frac{\partial \hat{\varepsilon}}{\partial \eta}>0 .
\end{aligned}
$$


Now (2.9) implies that (2.7) is invertible for $\eta$ as a function of $\mathbf{F}$ and $\varepsilon$, so that the constitutive expression can be rewritten in the entropic form $(\{\mathbf{F}, \varepsilon\}$ formulation) and the equation of state can be written as

$$
\eta=\hat{\eta}(\mathbf{F}, \varepsilon)
$$

In this formulation, the Cauchy stress $\mathbf{T}$ and the temperature $\theta$ are determined by

$$
\begin{aligned}
& \mathbf{T}=-\rho\left(\frac{\partial \hat{\eta}}{\partial \varepsilon}\right)^{-1} \frac{\partial \hat{\eta}}{\partial \mathbf{F}} \mathbf{F}^{T} \\
& \theta=\left(\frac{\partial \hat{\eta}}{\partial \varepsilon}\right)^{-1}>0
\end{aligned}
$$

Equations (2.10), (2.11), and (2.12) are referred to as the entropic form of the equations of state or the $\{\mathbf{F}, \varepsilon\}$ formulation, where $\mathbf{F}$ and $\varepsilon$ are independent variables.

In thermoelasticity, the positive definitions of certain material parameters obtained from the second derivatives of the potential function are considered as a mark of stability (see Scott [9]). It is generally assumed that the specific heat at constant deformation and the bulk modulus must be positive for stability (see Scott [9] and Gunton and Saunders [6]). The specific heat at constant deformation $C_{v}$ and at constant stress $C_{p}$ is defined as (see, e.g., Scott [12])

$$
C_{v}=\theta\left(\frac{\partial \eta}{\partial \theta}\right)_{\text {fixed } \mathbf{F}}, \quad C_{p}=\theta\left(\frac{\partial \eta}{\partial \theta}\right)_{\text {fixed } \mathbf{T}}
$$

and, through mathematical manipulation (see Callen [2, Chapter 7]), $C_{v}$ is derived from constitutive expressions in the three different formulations as

$$
C_{v}=-\theta \frac{\partial^{2} \hat{\psi}}{\partial \theta^{2}}=\frac{\partial \hat{\varepsilon}}{\partial \eta}\left(\frac{\partial^{2} \hat{\varepsilon}}{\partial \eta^{2}}\right)^{-1}=-\left(\frac{\partial \hat{\eta}}{\partial \varepsilon}\right)^{2}\left(\frac{\partial^{2} \hat{\eta}}{\partial \varepsilon^{2}}\right)^{-1}
$$

Using Maxwell relations (see Callen [2, Chapter 7] and Scott [12]), the bulk modulus at constant temperature $K_{\theta}$ can be obtained as

$$
\begin{aligned}
K_{\theta} & =\rho_{0} \frac{\partial^{2} \hat{\psi}}{\partial J^{2}}=\rho_{0} \frac{\partial^{2} \hat{\varepsilon}}{\partial J^{2}}-\rho_{0}\left(\frac{\partial^{2} \hat{\varepsilon}}{\partial J \partial \eta}\right)^{2}\left(\frac{\partial^{2} \hat{\varepsilon}}{\partial \eta^{2}}\right)^{-1} \\
& =-\rho_{0}\left(\frac{\partial \hat{\eta}}{\partial \varepsilon}\right)^{-1} \frac{\partial^{2} \hat{\eta}}{\partial J^{2}}+\rho_{0}\left(\frac{\partial \hat{\eta}}{\partial \varepsilon}\right)^{-1}\left(\frac{\partial^{2} \hat{\eta}}{\partial \varepsilon \partial J}\right)^{2}\left(\frac{\partial^{2} \hat{\eta}}{\partial \varepsilon^{2}}\right)^{-1} .
\end{aligned}
$$

These results are tabulated in Table 2.1 for convenience. 
Table 2.1. Summary of constitutive relationship for unconstrained case.

\begin{tabular}{|c|c|c|c|}
\hline & $\begin{array}{l}\{\mathbf{F}, \theta\} \\
\text { formulation }\end{array}$ & $\begin{array}{l}\{\mathbf{F}, \eta\} \\
\text { formulation }\end{array}$ & $\begin{array}{l}\{\mathbf{F}, \varepsilon\} \\
\text { formulation }\end{array}$ \\
\hline $\begin{array}{l}\text { Equation } \\
\text { of state }\end{array}$ & $\psi=\hat{\psi}(\mathbf{F}, \theta)$ & $\varepsilon=\hat{\varepsilon}(\mathbf{F}, \eta)$ & $\eta=\hat{\eta}(\mathbf{F}, \varepsilon)$ \\
\hline $\begin{array}{l}\text { Dependent } \\
\text { variables }\end{array}$ & $\begin{array}{l}\mathbf{T}=\rho \frac{\partial \hat{\psi}}{\partial \mathbf{F}} \mathbf{F}^{T} \\
\eta=-\frac{\partial \hat{\psi}}{\partial \theta}\end{array}$ & $\begin{array}{l}\mathbf{T}=\rho \frac{\partial \hat{\varepsilon}}{\partial \mathbf{F}} \mathbf{F}^{T} \\
\theta=\frac{\partial \hat{\varepsilon}}{\partial \eta}\end{array}$ & $\begin{array}{l}\mathbf{T}=-\rho\left(\frac{\partial \hat{\eta}}{\partial \varepsilon}\right)^{-1} \frac{\partial \hat{\eta}}{\partial \mathbf{F}} \mathbf{F}^{T} \\
\theta=\left(\frac{\partial \hat{\eta}}{\partial \varepsilon}\right)^{-1}\end{array}$ \\
\hline Specific heat & $C_{v}=-\theta \frac{\partial^{2} \psi}{\partial \theta^{2}}$ & $C_{v}=\frac{\partial \varepsilon}{\partial \eta}\left(\frac{\partial^{2} \varepsilon}{\partial \eta^{2}}\right)^{-1}$ & $C_{v}=-\left(\frac{\partial \eta}{\partial \varepsilon}\right)^{2}\left(\frac{\partial^{2} \eta}{\partial \varepsilon^{2}}\right)^{-1}$ \\
\hline Bulk modulus & $K_{\theta}=\rho_{0} \frac{\partial^{2} \hat{\psi}}{\partial J^{2}}$ & $\begin{aligned} K_{\theta}= & \rho_{0} \frac{\partial^{2} \hat{\varepsilon}}{\partial J^{2}} \\
& -\rho_{0}\left(\frac{\partial^{2} \hat{\varepsilon}}{\partial J \partial \eta}\right)^{2}\left(\frac{\partial^{2} \hat{\varepsilon}}{\partial \eta^{2}}\right)^{-1}\end{aligned}$ & $\begin{aligned} K_{\theta}= & -\rho_{0}\left(\frac{\partial \hat{\eta}}{\partial \varepsilon}\right)^{-1} \frac{\partial^{2} \hat{\eta}}{\partial J^{2}} \\
& +\rho_{0}\left(\frac{\partial \hat{\eta}}{\partial \varepsilon}\right)^{-1}\left(\frac{\partial^{2} \hat{\eta}}{\partial \varepsilon \partial J}\right)^{2}\left(\frac{\partial^{2} \hat{\eta}}{\partial \varepsilon^{2}}\right)^{-1}\end{aligned}$ \\
\hline
\end{tabular}

\section{Thermoelasticity with constraints}

In a general elastic material, it is clear that the entropy $\eta$ given by (2.5) is a function of both deformation gradient and temperature. Many polymeric materials are characterized by the assumption that the entropy $\eta$ is given by

$$
\eta=\tilde{\eta}(\mathbf{F})
$$

Usually, in the $\{\mathbf{F}, \theta\}$ formulation, this is treated as a constitutive assumption in the sense that (2.5) and (3.1) imply that $\psi$ must be affine in $\theta$. However, in the $\{\mathbf{F}, \eta\}$ formulation, such as that used here and by Scott $[11,12], \mathbf{F}$ and $\eta$ are the independent variables so that (3.1) may be considered as a specific case of a general deformation-entropy constraint of the form

$$
f(\mathbf{F}, \eta)=0 .
$$

In other words, the relationship (3.1) can be viewed in two different ways: (i) as special constitutive assumption obtained from a Helmholtz potential in the classical $\{\mathbf{F}, \theta\}$ formulation where $\eta$ is a derived quantity, or (ii) as a special case of an entropy-deformation constraint (3.2) in the $\{\mathbf{F}, \eta\}$ formulation. In the former case, there is no need for the introduction of a constraint response whereas the same equation (3.1) would require a constraint response in the $\{\mathbf{F}, \eta\}$ formulation. It is clear that final differential equations must be the same, no matter which formulation is used. We will explore this issue in more detail.

In order to investigate the effect of constraints in a thermoelastic body, we will consider three classes of constraints: (a) deformation-temperature constraints, (b) deformationentropy constraints, and (c) deformation-energy constraints. The different formulations 
of the basic constitutive relations are specifically suited to investigate specific classes of constraints.

3.1. Deformation-temperature constraint as a limit of an unconstrained body. We first consider the $\{\mathbf{F}, \theta\}$ formulation and consider a constraint relating the deformation gradient and temperature in the form

$$
\theta=\mathscr{T}(\mathbf{F})
$$

Such a constraint was originally proposed as a generalization of a purely mechanical constraint, such as incompressibility, to allow for thermal expansion but retain incompressibility under isothermal conditions.

As Scott [11] in the context of thermoelasticity and the authors [1] in the context of diffusion showed, rather than imposing the constraints directly via Lagrange multipliers, considering these constraints as limiting cases helps to understand the behavior of the material and to provide a physical meaning to the constraint response.

Thus, consider an unconstrained thermoelastic material and introduce an auxiliary variable $\phi$ as

$$
\phi=\theta-\mathscr{T}(\mathbf{F})
$$

Instead of augmenting the Helmholtz potential energy for the constrained material (e.g., Scott [12] used the form $\left.\psi^{*}=\psi+(1 / 2) \chi \phi^{2}\right)$, we can choose $\mathbf{F}$ and $\phi$ as independent variables instead of $\mathbf{F}$ and $\theta$ for the Helmholtz potential function. Using (3.4), the equation of state (2.3) for an unconstrained material can be written in the form

$$
\psi=\hat{\psi}(\mathbf{F}, \theta)=\hat{\psi}(\mathbf{F}, \phi+\mathscr{T}(\mathbf{F}))=\tilde{\psi}(\mathbf{F}, \phi) .
$$

Using the chain rule, the entropy $\eta$ becomes

$$
\eta=-\frac{\partial \psi}{\partial \theta}=-\frac{\partial \tilde{\psi}}{\partial \phi}
$$

and the Cauchy stress $\mathbf{T}$ is given by

$$
\mathbf{T}=\rho \frac{\partial \tilde{\psi}}{\partial \mathbf{F}} \mathbf{F}^{T}+\rho \eta \frac{\partial \mathcal{T}}{\partial \mathbf{F}} \mathbf{F}^{T}
$$

We now consider the limit when $\phi$ tends to zero. Expanding $\eta$ as a Taylor series around $\phi=0$,

$$
\eta(\phi)=-\left.\left(\frac{\partial \tilde{\psi}}{\partial \phi}\right)\right|_{\phi=0}-\left.\left(\frac{\partial^{2} \tilde{\psi}}{\partial \phi^{2}}\right)\right|_{\phi=0} \phi-\cdots,
$$

where $\partial \tilde{\psi} / \partial \phi$ is a finite value at $\phi=0$. In the limit, we assume that $\partial^{2} \tilde{\psi} /\left.\partial \phi^{2}\right|_{\phi=0} \rightarrow \pm \infty$ and $\phi \rightarrow 0$ in such a way that their product remains finite. It is also assumed that higher-order terms in (3.8) are successively of smaller orders of magnitude and give no contribution to $\eta$ in the limit. So the sum of the terms in (3.8) yields an arbitrary but finite value for $\eta$. 
Thus in the limit, the entropy becomes an independent variable and plays the role of a Lagrange multiplier whereas the temperature is given by (3.3), although for the unconstrained situation, the temperature is the independent variable and the entropy is a derived quantity. This limiting procedure also implies that in the limit as $\phi \rightarrow 0$, the Helmholtz potential becomes a function of the deformation gradient $\mathbf{F}$ alone, that is,

$$
\lim _{\phi \rightarrow 0} \tilde{\psi}(\mathbf{F}, \phi)=\tilde{\psi}(\mathbf{F}, 0)=\mathscr{F}(\mathbf{F}) .
$$

Thus, the stress reduces to

$$
\mathbf{T}=\rho \frac{\partial \mathscr{F}(\mathbf{F})}{\partial \mathbf{F}} \mathbf{F}^{T}+\rho \eta \frac{\partial \mathscr{T}(\mathbf{F})}{\partial \mathbf{F}} \mathbf{F}^{T}
$$

where $\eta$ is arbitrary and finite.

As we mentioned before, the positiveness of $C_{v}$ and $K_{\theta}$ is desirable for the stability of the body and the sign of $\partial^{2} \tilde{\psi} /\left.\partial \phi^{2}\right|_{\phi=0}$ is critical in deciding the sign of the second derivatives of the Helmholtz potential. First, let $\partial^{2} \tilde{\psi} /\left.\partial \phi^{2}\right|_{\phi=0} \rightarrow-\infty$. Then, the specific heat $C_{v}$ defined by (2.14) becomes positive infinity in the limit, as seen by expanding (2.14):

$$
C_{v}=-\left.\mathscr{T}(\mathbf{F})\left(\frac{\partial^{2} \tilde{\psi}}{\partial \phi^{2}}\right)\right|_{\phi=0}-\left.\mathscr{T}(\mathbf{F})\left(\frac{\partial^{3} \tilde{\psi}}{\partial \phi^{3}}\right)\right|_{\phi=0} \phi \cdots \longrightarrow \infty
$$

Using the chain rule, the bulk modulus at the constant temperature $K_{\theta}$ defined in (2.15) is expanded as

$$
K_{\theta}=\rho_{0} \frac{\partial^{2} \hat{\psi}}{\partial J^{2}}=\left.\rho_{0}\left\{\frac{\partial^{2} \tilde{\psi}}{\partial J^{2}}-2 \frac{\partial^{2} \tilde{\psi}}{\partial J \partial \phi} \frac{\partial \mathscr{T}}{\partial J}-\frac{\partial \tilde{\psi}}{\partial \phi} \frac{\partial^{2} \mathscr{T}}{\partial J^{2}}+\frac{\partial^{2} \tilde{\psi}}{\partial \phi^{2}}\left(\frac{\partial \mathscr{T}}{\partial J}\right)^{2}\right\}\right|_{\phi=0}+\cdots \cdot
$$

In the limit as $\phi \rightarrow 0$ and $\partial^{2} \tilde{\psi} /\left.\partial \phi^{2}\right|_{\phi=0} \rightarrow-\infty$, assuming that the first derivative of $\tilde{\psi}$ with respect to $\phi$ and the derivatives of $\mathscr{T}$ in (3.12) are finite, the bulk modulus $K_{\theta}$ goes to negative infinity. Thus, the assumption $\partial^{2} \tilde{\psi} /\left.\partial \phi^{2}\right|_{\phi=0} \rightarrow-\infty$ implies that the specific heat at constant deformation tends to $+\infty$ but isothermal bulk modulus tends to $-\infty$, which implies the loss of the stability of the body.

Next, if we assume that $\partial^{2} \tilde{\psi} /\left.\partial \phi^{2}\right|_{\phi=0} \rightarrow \infty$ in the limit as $\phi \rightarrow 0$, the isothermal bulk modulus goes to positive infinity, but the specific heat at constant deformation becomes negative infinity. This is again physically unacceptable.

Thus the limiting process reveals that, although the deformation temperature constraint appears to be a straight forward generalization of a constraint such as incompressibility, it results in the material becoming unstable.

It is instructive to note that the same constitutive equations may be obtained without the use of a limiting process in the $\{\mathbf{F}, \eta\}$ formulation. In this case, condition (3.3) can be simply inferred by using the constitutive form

$$
\varepsilon=\hat{\varepsilon}(\mathbf{F}, \eta)=\mathscr{F}(\mathbf{F})+\eta \mathscr{T}(\mathbf{F}) .
$$


Using (2.9), the energy function (3.13) immediately gives (3.3). But now, (3.3) is viewed as a constitutive equation. Furthermore, the stress results in (3.10). Also, substituting (3.13) into the energetic formulation in (2.14) and (2.15), we can easily see that $C_{v}=\infty$ and $K_{\theta}=-\infty$.

3.2. Deformation-entropy constraint as a limit of an unconstrained body. We consider the $\{\mathbf{F}, \eta\}$ formulation and a different generalization of the purely mechanical constraint in the form $f(\mathbf{F}, \eta)=0$. Under the assumption that $f$ is monotonically increasing function for $\eta$, that is,

$$
\frac{\partial f}{\partial \eta}>0
$$

the constraint can be rewritten in the form

$$
\eta=\mathscr{S}(\mathbf{F})
$$

Scott [11] has introduced a linearized form of (3.15) connecting the deformation and the entropy as an alternative to the temperature-deformation constraint (3.3).

In this case, the variable $\phi$ introduced in (3.4) is replaced by

$$
\phi=\eta-\mathscr{Y}(\mathbf{F}) .
$$

Using (3.16), the constitutive equation (2.7) for the internal energy of an unconstrained material can be rewritten in the form

$$
\varepsilon=\hat{\varepsilon}(\mathbf{F}, \eta)=\tilde{\varepsilon}(\mathbf{F}, \phi) .
$$

Using the chain rule, it can be shown that $\partial \tilde{\mathcal{E}} / \partial \phi$ is the absolute temperature, that is,

$$
\theta=\frac{\partial \varepsilon}{\partial \eta}=\frac{\partial \tilde{\varepsilon}}{\partial \phi}
$$

and the Cauchy stress $\mathbf{T}$ now becomes

$$
\mathbf{T}=\rho \frac{\partial \tilde{\varepsilon}}{\partial \mathbf{F}} \mathbf{F}^{T}-\rho \theta \frac{\partial \mathscr{S}}{\partial \mathbf{F}} \mathbf{F}^{T}
$$

where $\theta$ plays the role of the Lagrange multiplier.

We assume that, in the limit as $\phi \rightarrow 0,\left.(\partial \tilde{\varepsilon} / \partial \phi)\right|_{\phi=0}$ is a finite value, and the product of $\partial^{2} \tilde{\varepsilon} /\left.\partial \phi^{2}\right|_{\phi=0} \rightarrow \infty$ and $\phi$ is arbitrary and finite. Under these conditions, the function $\theta$ in (3.18) can be expanded as a Taylor series around $\phi=0$ as

$$
\theta(\phi)=\left.\frac{\partial \tilde{\varepsilon}}{\partial \phi}\right|_{\phi=0}+\left.\frac{\partial^{2} \tilde{\varepsilon}}{\partial \phi^{2}}\right|_{\phi=0} \phi+\cdots
$$

Higher-order terms in (3.20) are smaller orders of magnitude so that the sum of the terms in (3.20) yields an arbitrary but finite value. In the limit, (3.17) reduces to

$$
\lim _{\phi \rightarrow 0} \tilde{\varepsilon}(\mathbf{F}, \phi)=q(\mathbf{F}) .
$$


Thus, the constraint $\eta=\mathscr{S}(\mathbf{F})$ implies that $\varepsilon=\mathscr{U}(\mathbf{F})$ and the stress is rewritten as

$$
\mathbf{T}=\rho \frac{\partial \mathcal{U}(\mathbf{F})}{\partial \mathbf{F}} \mathbf{F}^{T}-\rho \theta \frac{\partial \mathscr{S}(\mathbf{F})}{\partial \mathbf{F}} \mathbf{F}^{T}
$$

We observe from (3.22) that the stress response is linear with respect to the temperature $\theta$.

From the conditions for the limit as $\phi \rightarrow 0$ and $\partial^{2} \tilde{\varepsilon} /\left.\partial \phi^{2}\right|_{\phi=0} \rightarrow \pm \infty$, the specific heat $C_{v}$ goes to zero by expanding (2.14) as a Taylor series around $\phi=0$ as

$$
C_{v}=\left.\frac{\partial \tilde{\varepsilon}}{\partial \phi}\left(\frac{\partial^{2} \tilde{\varepsilon}}{\partial \phi^{2}}\right)^{-1}\right|_{\phi=0}+\left.\left(1-\frac{\partial \tilde{\varepsilon}}{\partial \phi} \frac{\partial^{3} \tilde{\varepsilon}}{\partial \phi^{3}}\left(\frac{\partial^{2} \tilde{\varepsilon}}{\partial \phi^{2}}\right)^{-2}\right)\right|_{\phi=0} \phi+\cdots \longrightarrow 0
$$

Using the chain rule, the bulk modulus at constant temperature $K_{\theta}$ given in (2.15) can be rewritten as

$$
\begin{aligned}
K_{\theta}= & \rho_{0}\left(\frac{\partial^{2} \tilde{\varepsilon}}{\partial J^{2}}-2 \frac{\partial^{2} \tilde{\varepsilon}}{\partial J \partial \phi} \frac{\partial \mathscr{S}}{\partial J}-\frac{\partial \tilde{\varepsilon}}{\partial \phi} \frac{\partial^{2} \mathscr{S}}{\partial J^{2}}+\frac{\partial^{2} \tilde{\varepsilon}}{\partial \phi^{2}}\left(\frac{\partial \mathscr{S}}{\partial J}\right)^{2}\right) \\
& -\rho_{0}\left(\frac{\partial^{2} \tilde{\varepsilon}}{\partial \phi \partial J}-\frac{\partial^{2} \tilde{\varepsilon}}{\partial \phi^{2}} \frac{\partial \mathscr{S}}{\partial J}\right)^{2}\left(\frac{\partial^{2} \tilde{\varepsilon}}{\partial \phi^{2}}\right) \\
= & \rho_{0}\left\{\frac{\partial^{2} \tilde{\varepsilon}}{\partial J^{2}}-\frac{\partial \tilde{\varepsilon}}{\partial \phi} \frac{\partial^{2} \mathscr{S}}{\partial J^{2}}-\left(\frac{\partial \tilde{\varepsilon}}{\partial \phi \partial J}\right)^{2}\left(\frac{\partial^{2} \tilde{\varepsilon}}{\partial \phi^{2}}\right)^{-1}\right\} .
\end{aligned}
$$

In the limit as $\phi \rightarrow 0$, the value of $\partial^{2} \tilde{\varepsilon} /\left.\partial \phi^{2}\right|_{\phi=0}$ goes to $\pm \infty$ so that the last term of the right-hand side of (3.24) vanishes. Finally, in the limit, $K_{\theta}$ is reduced to

$$
K_{\theta}=\left.\rho_{0}\left(\frac{\partial^{2} \tilde{\varepsilon}}{\partial J^{2}}-\theta \frac{\partial^{2} \mathscr{S}}{\partial J^{2}}\right)\right|_{\phi=0}+\cdots
$$

where $\theta$ is arbitrary and finite.

Thus, in the limit as $\phi \rightarrow 0$ and $\partial^{2} \tilde{\varepsilon} /\left.\partial \phi^{2}\right|_{\phi=0} \rightarrow \pm \infty, C_{v}$ goes to zero and the condition for the positive $K_{\theta}$ becomes

$$
\frac{\partial^{2} \tilde{\varepsilon}}{\partial J^{2}}-\theta \frac{\partial^{2} \mathscr{S}}{\partial J^{2}}>0
$$

Similar to the case of the deformation-temperature constraint, (3.15), which is given as the deformation-entropy constraint in the $\{\mathbf{F}, \eta\}$ formulation, can be obtained as a constitutive relation derived from the Helmholtz potential form of the equation of state of the form

$$
\psi(\mathbf{F}, \theta)=\vartheta(\mathbf{F})-\theta \mathscr{S}(\mathbf{F}) .
$$


Substituting (3.27) into (2.4), the stress $\mathbf{T}$ is derived the same as (3.22). Also, substituting (3.27) into the formulations for the Helmh potential in (2.14) and (2.15), the specific heat $C_{v}$ and the bulk modulus $K_{\theta}$ are derived as

$$
\begin{gathered}
C_{v}=0, \\
K_{\theta}=\rho_{0} \frac{\partial^{2} U}{\partial J^{2}}-\rho_{0} \theta \frac{\partial^{2} \mathscr{S}}{\partial J^{2}} .
\end{gathered}
$$

In this formulation, the temperature is the independent variable.

3.3. Deformation-energy constraint as a limit of an unconstrained body. In Section 3.2, we saw from (3.21) that the deformation-entropy constraint (3.15) implies that the internal energy is a function of the deformation gradient only. Now, we consider the deformation-energy constraint in the $\{\mathbf{F}, \varepsilon\}$ formulation in the form

$$
\varepsilon=U(\mathbf{F})
$$

Then, the auxiliary variable $\phi$ is written as

$$
\phi=\varepsilon-\vartheta(\mathbf{F})
$$

and $\phi$ goes to zero when (3.29) holds. Using (3.30), the equation of state (2.10) of an unconstrained material can be written in the form

$$
\eta=\eta(\mathbf{F}, \varepsilon)=\tilde{\eta}(\mathbf{F}, \phi)
$$

Using (2.11) and the chain rule, the Cauchy stress $\mathbf{T}$ becomes

$$
\mathbf{T}=-\rho\left(\frac{\partial \tilde{\eta}}{\partial \phi}\right)^{-1}\left(\frac{\partial \tilde{\eta}}{\partial \mathbf{F}} \mathbf{F}^{T}-\frac{\partial \tilde{\eta}}{\partial \phi} \frac{\partial \boldsymbol{U}}{\partial \mathbf{F}} \mathbf{F}^{T}\right)=\rho \frac{\partial \boldsymbol{U}}{\partial \mathbf{F}} \mathbf{F}^{T}-\rho\left(\frac{\partial \tilde{\eta}}{\partial \phi}\right)^{-1} \frac{\partial \tilde{\eta}}{\partial \mathbf{F}} \mathbf{F}^{T}
$$

We also see that

$$
\theta=\left(\frac{\partial \eta}{\partial \varepsilon}\right)^{-1}=\left(\frac{\partial \tilde{\eta}}{\partial \phi}\right)^{-1}
$$

Now we assume that $\left.(\partial \tilde{\eta} / \partial \phi)^{-1}\right|_{\phi=0}$ is a finite value and $\partial^{2} \tilde{\eta} /\left.\partial \phi^{2}\right|_{\phi=0} \rightarrow \infty$ so that the product of $\partial^{2} \tilde{\eta} / \partial \phi^{2}$ and $\phi$ becomes arbitrary and finite at the limit. Under these conditions, $\theta$ can be expanded as a Taylor series around $\phi=0$ as

$$
\theta(\phi)=\left.\left(\frac{\partial \tilde{\eta}}{\partial \phi}\right)^{-1}\right|_{\phi=0}-\frac{\partial^{2} \tilde{\eta}}{\partial \phi^{2}}\left(\frac{\partial \tilde{\eta}}{\partial \phi}\right)_{\mid \phi=0}^{-2} \phi+\cdots,
$$

where higher-order terms in (3.34) are smaller orders on magnitude. So the sum of the terms in (3.34) yields an arbitrary but finite value. 
At the limit, the entropy becomes a function of $\mathbf{F}$ alone:

$$
\lim _{\phi \rightarrow 0} \tilde{\eta}(\mathbf{F}, \phi)=\mathscr{S}(\mathbf{F})
$$

Substituting (3.35) into (3.32), the stress $\mathbf{T}$ reduces to the same as (3.22). Here again $\theta$ plays the role of the Lagrange multiplier. From the conditions for the limit as $\phi \rightarrow 0$ and $\partial^{2} \tilde{\eta} /\left.\partial \phi^{2}\right|_{\phi=0} \rightarrow-\infty$, the specific heat $C_{v}$ goes to zero by expanding (2.14) as a Taylor series around $\phi=0$ as

$$
\begin{aligned}
C_{v}= & -\left.\left(\frac{\partial \tilde{\eta}}{\partial \phi}\right)^{2}\left(\frac{\partial^{2} \tilde{\eta}}{\partial \phi^{2}}\right)^{-1}\right|_{\phi=0} \\
& -\left.\left(2 \frac{\partial \tilde{\eta}}{\partial \phi}\left(\frac{\partial^{2} \tilde{\eta}}{\partial \phi^{2}}\right)^{-1}-\frac{\partial^{3} \tilde{\eta}}{\partial \phi^{3}}\left(\frac{\partial \tilde{\eta}}{\partial \phi}\right)^{2}\left(\frac{\partial^{2} \tilde{\eta}}{\partial \phi^{2}}\right)^{-2}\right)\right|_{\phi=0} \phi-\cdots \longrightarrow 0 .
\end{aligned}
$$

Using the chain rule, $K_{\theta}$ in (2.15) can be rewritten as

$$
\begin{aligned}
K_{\theta}= & -\rho_{0}\left(\frac{\partial \tilde{\eta}}{\partial \phi}\right)^{-1}\left(\frac{\partial^{2} \tilde{\eta}}{\partial J^{2}}-2 \frac{\partial^{2} \tilde{\eta}}{\partial J \partial} \frac{\partial \mathcal{U}}{\partial J}-\frac{\partial \tilde{\eta}}{\partial \phi} \frac{\partial^{2} U}{\partial J^{2}}+\frac{\partial^{2} \tilde{\eta}}{\partial \phi^{2}}\left(\frac{\partial^{\mathcal{U}}}{\partial J}\right)^{2}\right) \\
& +\rho_{0}\left(\frac{\partial \tilde{\eta}}{\partial \phi}\right)^{-1}\left(\frac{\partial^{2} \tilde{\eta}}{\partial \phi \partial J}-\frac{\partial^{2} \tilde{\eta}}{\partial \phi^{2}} \frac{\partial \mathcal{U}}{\partial J}\right)^{2}\left(\frac{\partial^{2} \tilde{\eta}}{\partial \phi^{2}}\right)^{-1} \\
= & -\rho_{0}\left(\frac{\partial \tilde{\eta}}{\partial \phi}\right)^{-1}\left\{\frac{\partial^{2} \tilde{\eta}}{\partial J^{2}}-\frac{\partial \tilde{\eta}}{\partial \phi} \frac{\partial^{2} U}{\partial J^{2}}-\left(\frac{\partial^{2} \tilde{\eta}}{\partial \phi \partial J}\right)^{2}\left(\frac{\partial^{2} \tilde{\eta}}{\partial \phi^{2}}\right)^{-1}\right\} .
\end{aligned}
$$

In the limit as $\phi \rightarrow 0$, the value of $\partial^{2} \tilde{\eta} /\left.\partial \phi^{2}\right|_{\phi=0}$ goes to $\pm \infty$ so that the last term of the right-hand side of (3.37) vanishes. Finally, in the limit, $K_{\theta}$ is reduced to

$$
K_{\theta}=\left.\rho_{0}\left(\frac{\partial^{2} u}{\partial J^{2}}-\theta \frac{\partial^{2} \tilde{\eta}}{\partial J^{2}}\right)\right|_{\phi=0}+\cdots
$$

where $\theta$ is arbitrary and finite. Thus, in the limit as $\phi \rightarrow 0$ and $\partial^{2} \tilde{\eta} /\left.\partial \phi^{2}\right|_{\phi=0} \rightarrow \pm \infty, C_{v}$ goes to zero and the condition for the positive $K_{\theta}$ becomes

$$
\frac{\partial^{2} u}{\partial J^{2}}-\theta \frac{\partial^{2} \tilde{\eta}}{\partial J^{2}}>0
$$

From Sections 3.2 and 3.3, the deformation-entropy constraint, $\eta=\mathscr{Y}(\mathbf{F})$, implies that the constitutive form for the internal energy is a function of deformation alone, that is, $\eta=\mathscr{S}(\mathbf{F}) \Rightarrow \varepsilon=\mathcal{\varepsilon}(\mathbf{F})$, and the deformation-energy constraint $\varepsilon=\mathcal{U}(\mathbf{F})$ implies that the entropy is a function of the deformation gradient alone. Thus, we see that

$$
\eta=\mathscr{S}(\mathbf{F}) \Longleftrightarrow \varepsilon=\mathcal{U}(\mathbf{F}),
$$


164 Thermoelasticity with constraints

Table 3.1. Thermomechanical constraints and equivalent forms of the thermodynamic potentials.

\begin{tabular}{|c|c|c|}
\hline \multirow{2}{*}{ Constraint form } & $\varepsilon=U(\mathbf{F})$ & $\theta=\mathscr{T}(\mathbf{F})$ \\
\hline & $\eta=\mathscr{S}(\mathbf{F})$ & $\psi=\mathscr{F}(\mathbf{F})$ \\
\hline Equivalent form & $\psi(\mathbf{F}, \theta)=U(\mathbf{F})-\theta \mathscr{S}(\mathbf{F})$ & $\varepsilon(\mathbf{F}, \eta)=\mathscr{F}(\mathbf{F})+\eta \mathscr{T}(\mathbf{F})$ \\
\hline Stress & $\begin{array}{c}\mathbf{T}=\rho \frac{\partial \boldsymbol{U}}{\partial \mathbf{F}} \mathbf{F}^{T}-\rho \theta \frac{\partial \mathscr{S}}{\partial \mathbf{F}} \mathbf{F}^{T} \\
\theta \text { independent }\end{array}$ & $\begin{aligned} \mathbf{T}= & \rho \frac{\partial \mathscr{F}_{F}}{\partial \mathbf{F}} \mathbf{F}^{T}+\rho \eta \frac{\partial \mathscr{T}}{\partial \mathbf{F}} \mathbf{F}^{T} \\
& \eta \text { independent }\end{aligned}$ \\
\hline Specific heat & $C_{v}=0$ & $C_{v}=\infty$ \\
\hline Bulk modulus & $K_{\theta}=\rho_{0} \frac{\partial^{2} U}{\partial J^{2}}-\rho_{0} \theta \frac{\partial^{2} \varphi}{\partial J^{2}}$ & $K_{\theta}=-\infty$ \\
\hline
\end{tabular}

and in both cases the absolute temperature plays the role of the Lagrange multiplier. It is interesting to note that both constraints (3.15) and (3.29) can be implemented by a special Helmholtz potential of the form

$$
\psi(\mathbf{F}, \theta)=\vartheta(\mathbf{F})-\theta \mathscr{S}(\mathbf{F})
$$

The stress response derived from (3.41) is identical to (3.22) and the specific heat turns out to be zero.

Moreover, as Scott [12] found, we can obtain the same constitutive responses as those from the deformation-entropy and deformation-energy constraints if we simply require that $C_{v}=0$. To see this, substituting $C_{v}=0$ into (2.13), we obtain that

$$
\left(\frac{\partial \eta}{\partial \theta}\right)_{\text {fixed } F}=0
$$

which implies that the entropy is a function of $\mathbf{F}$ only. Also substituting $C_{v}=0$ into (2.14) results in

$$
\frac{\partial^{2} \hat{\psi}}{\partial \theta^{2}}=0
$$

which implies that the Helmholtz potential is affine with respect to the temperature. Thus, simply setting $C_{v}=0$ in a Helmholtz potential is equivalent to the deformationentropy and deformation-energy constraints.

Therefore the following four assumption are equivalent:

(1) $\eta=\mathscr{S}(\mathbf{F})$

(2) $\varepsilon=U(\mathbf{F})$

(3) $\psi=\vartheta(\mathbf{F})-\theta \mathscr{S}(\mathbf{F})$,

(4) $C_{v}=0$.

We summarize the thermomechanical constraints and the equivalent forms of potential functions in Table 3.1. 


\section{The application of thermomechanical constraint to a rubberlike material}

In polymeric materials, statistical mechanics considerations reveal that the entropy is significantly affected by isochoric deformation whereas the internal energy hardly changes so that a simple incompressible thermoelastic model for polymeric solids assumes that (1) the material is incompressible, (2) $\eta=\hat{\eta}(\mathbf{F})$, and (3) the internal energy is constant. Such a model is usually referred to as purely entropic elasticity.

It has been shown by Chadwick [3] that such an idealization is inadequate for the realistic modeling of thermoelastic processes. Motivated by the results of Section 3, we consider a generalization of the above questions by assuming that (a) the material is compressible and (b) the energy $\varepsilon$ is a function of $J$ alone. Note that assumption (b) implies that, for isochoric deformations, the energy is constant in keeping up with the idealizations used in purely entropic elasticity. In view of the results in Section 3, assumption (b) precludes the entropy $\eta$ from being a function of the temperature so that

$$
\eta=\mathscr{S}(\mathbf{F})
$$

Furthermore, the assumption of the energy implies that the Helmholtz potential is of the form

$$
\psi=-\theta \mathscr{S}(\mathbf{F})+\varepsilon(J)
$$

A specific form for $\mathscr{S}(\mathbf{F})$ and $\varepsilon(J)$ can be derived from the constitutive equations which were suggested by Chadwick [3] by letting the specific heat $C_{v} \rightarrow 0$. To elaborate, Chadwick considered a Helmholtz potential of the form

$$
\psi(\mathbf{F}, \theta)=\frac{\theta}{\theta_{0}}\left[\psi_{0}(\mathbf{F})-\varepsilon_{0}(\mathbf{F})\right]+\varepsilon_{0}(\mathbf{F})-A\left(\theta \ln \frac{\theta}{\theta_{0}}-\theta+\theta_{0}\right) .
$$

The stress T, obtained by combining (2.4) and (4.3), is given by

$$
\mathbf{T}=\rho \frac{\theta}{\theta_{0}} \frac{\partial \psi_{0}}{\partial \mathbf{F}} \mathbf{F}^{T}+\rho_{0}\left(1-\frac{\theta}{\theta_{0}}\right) \frac{\partial \varepsilon_{0}}{\partial J} \mathbf{I},
$$

and $\eta$ is given by

$$
\eta(\mathbf{F}, \theta)=-\frac{1}{\theta_{0}}\left[\psi_{0}(\mathbf{F})-\varepsilon_{0}(J)\right]+A \ln \frac{\theta}{\theta_{0}} .
$$

The entropy $\eta$ is separated into two parts: the first part is a function of the deformation gradient and the second part is a function of the temperature alone. The specific heat at constant deformation $C_{v}$ and $C_{p}$ at constant stress $\mathbf{T}=\mathbf{T}^{*}$ is given by

$$
\begin{gathered}
C_{v}=A \\
C_{p}=C_{v}+\theta \frac{\partial \eta}{\partial J}\left(\frac{\partial J}{\partial \theta}\right)_{\mathrm{T}=\mathrm{T}^{*}} .
\end{gathered}
$$

We also note that the value of $C_{v}$ has no effect on the stress response. 
4.1. Application of deformation-entropy and deformation-energy constraints. In view of Section 3, the equivalent form of Helmholtz potential to the deformation-energy constraint can be obtained by setting $C_{v}=0$, which in this case reduces to $A=0$. Thus, letting $C_{v} \rightarrow 0$, the Helmholtz potential reduces to

$$
\psi(\mathbf{F}, \theta)=\frac{\theta}{\theta_{0}}\left[\psi_{0}(\mathbf{F})-\varepsilon_{0}(J)\right]+\varepsilon_{0}(J)
$$

Comparing (4.7) with (4.2), the equivalent constraints are obtained as

$$
\begin{gathered}
\varepsilon=\mathscr{U}(\mathbf{F})=\varepsilon_{0}(J), \\
\eta=\mathscr{Y}(\mathbf{F})=-\frac{1}{\theta_{0}}\left[\psi_{0}(\mathbf{F})-\varepsilon_{0}(J)\right] .
\end{gathered}
$$

Using (3.22), the stress is obtained as (4.4) in the unconstrained case. The specific heat $C_{p}$ is given by

$$
C_{p}=\theta \frac{\partial \eta}{\partial J}\left(\frac{\partial J}{\partial \theta}\right)_{\mathrm{T}=\mathbf{T}^{*}}
$$

We now consider empirical functions used by Chadwick [3] of the form

$$
\begin{gathered}
\psi_{0}(\mathbf{F})=\frac{\mu}{\rho_{0}}\left(I_{1}-3 J^{2 / 3}\right)+\frac{\kappa}{\rho_{0} m}\left(J+\frac{1}{m-1} J^{-m+1}-\frac{m}{m-1}\right), \\
\varepsilon_{0}(\mathbf{F})=\frac{\kappa \alpha \theta_{0}}{\rho_{0} n}\left(J^{n}-1\right) .
\end{gathered}
$$

We use thermoelastic material parameters of a peroxide-cured vulcanizate of natural rubber (see Chadwick [3] and Wood and Martin [14]) and they are the density $\rho_{0}=$ $906.5 \mathrm{~kg} / \mathrm{m}^{3}$, the shear modulus $\mu=4.2 \times 10^{2} \mathrm{kPa}$, the isothermal bulk modulus $\kappa=$ $1.95 \times 10^{6} \mathrm{kPa}$, the volume coefficient of thermal expansion $\alpha=6.36 \times 10^{-4} \mathrm{~K}^{-1}$, and the material parameters $m=9$ and $n=5 / 2$. The initial temperature $\theta_{0}$ is $25^{\circ} \mathrm{C}$.

In order to compare the numerical results of the constrained case with those of the unconstrained case, we consider a simple homogeneous deformation of the form

$$
x=J^{1 / 3} \lambda X, \quad y=\frac{J^{1 / 3}}{\sqrt{\lambda}} Y, \quad z=\frac{J^{1 / 3}}{\sqrt{\lambda}} Z,
$$

where $(X, Y, Z)$ are the position in reference configuration and $(x, y, z)$ are the position in current configuration in Cartesian coordinate. The deformation gradient is calculated as

$$
\mathbf{F}=J^{1 / 3}\left[\begin{array}{ccc}
\lambda & 0 & 0 \\
0 & \frac{1}{\sqrt{\lambda}} & 0 \\
0 & 0 & \frac{1}{\sqrt{\lambda}}
\end{array}\right], \quad \mathbf{B}=J^{2 / 3}\left[\begin{array}{ccc}
\lambda^{2} & 0 & 0 \\
0 & \frac{1}{\lambda} & 0 \\
0 & 0 & \frac{1}{\lambda}
\end{array}\right]
$$


where $\lambda$ is the isochoric biaxial stretch. The invariants of $\mathbf{B}$ are given as

$$
I_{1}=J^{2 / 3}\left(\lambda^{2}+\frac{2}{\lambda}\right), \quad I_{2}=2 J^{4 / 3} \lambda, \quad I_{3}=J^{2} .
$$

Substituting (4.11) into (4.9), the deformation-entropy relation is obtained by

$$
\eta=-\frac{\kappa}{\rho_{0} \theta_{0}}\left[\frac{\mu}{\kappa}\left(I_{1}-3 J^{2 / 3}\right)+\frac{J}{m}+\frac{J^{-m+1}-m}{m(m-1)}-\frac{\alpha \theta_{0}}{n}\left(J^{n}-1\right)\right] .
$$

It follows from (4.4) that the stress $\mathbf{T}$ is given by

$$
\mathbf{T}=\frac{2 \mu \theta}{J \theta_{0}}\left(\mathbf{B}-J^{2 / 3} \mathbf{I}\right)+\frac{\kappa \theta}{m \theta_{0}}\left(1-J^{-m}\right) \mathbf{I}+\kappa \alpha \theta_{0} J^{n-1}\left(1-\frac{\theta}{\theta_{0}}\right) \mathbf{I} .
$$

The volume-pressure relation for pure volume expansion can be found by substituting $\lambda=1$ and $\mathbf{T}=-P \mathbf{I}$ into (4.16) to give

$$
-P=\frac{\kappa \theta}{m \theta_{0}}\left(1-J^{-m}\right)+\kappa \alpha \theta_{0} J^{n-1}\left(1-\frac{\theta}{\theta_{0}}\right) .
$$

Figure 4.1 shows the volume temperature curves for four different nondimensionalized pressures $P / \kappa$. From (4.17), the relation between temperature and volume change in isothermal process and at the stress-free state is obtained by

$$
\theta=\frac{-\kappa \alpha \theta_{0} J^{n-1}}{\left(\kappa / m \theta_{0}\right)\left(1-J^{-m}\right)-\kappa \alpha J^{n-1}} .
$$

The specific heat at constant pressure $C_{p}$ can be obtained from (4.10) and (4.16) for the fixed value of the stress $\mathbf{T}$ and $\lambda$. When $\mathbf{T}=0$ and $\lambda=1, \partial J /\left.\partial \theta\right|_{\mathbf{T}=\mathbf{0}}$ can be obtained from (4.18) and substituting the result into (4.10) gives $C_{p}$-temperature curve shown in Figure 4.2. The results are compared with the unconstrained case and the experimental data of Wood and Martin [14].

Since the stress responses in isothermal deformation are the same for both constrained and unconstrained cases (see (4.4) and (4.10)), assume an isentropic deformation and compare the stress and temperature responses for the two cases. In order to find temperature and stress responses in isentropic homogeneous extension for the constrained case, we substitute the value of $\lambda$ and $\eta=0$ into (4.15) and obtain $J$. Then, the temperature and the biaxial stress are obtained from (4.16) by assuming other stress components to be zero except the axial stress $T_{x x}$ which is set to $\sigma$.

The variation of temperature with respect to the axial stretch $\Lambda$ for the isentropic process is plotted in Figure 4.3, where the axial stretch is defined by

$$
\Lambda=\lambda\left(\frac{J}{J_{0}}\right)^{1 / 3} \text {. }
$$

The nondimensionalized temperature changes by about $1 \%$ for up to two times of axial stretch, and the absolute value of temperature changes by about $3^{\circ} \mathrm{C}$. The stress responses plotted in Figure 4.4 are very close for both the constrained and unconstrained cases. 


\section{Thermoelasticity with constraints}

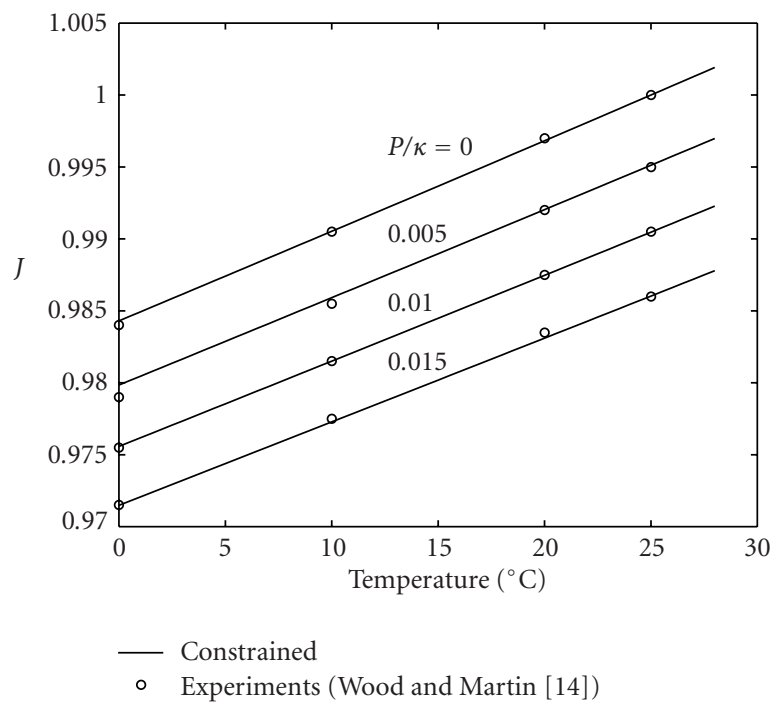

Figure 4.1. Volume expansion with temperature variation for different ambient pressures. Solid curves are for the constrained material. Experimental data is from Wood and Martin [14].

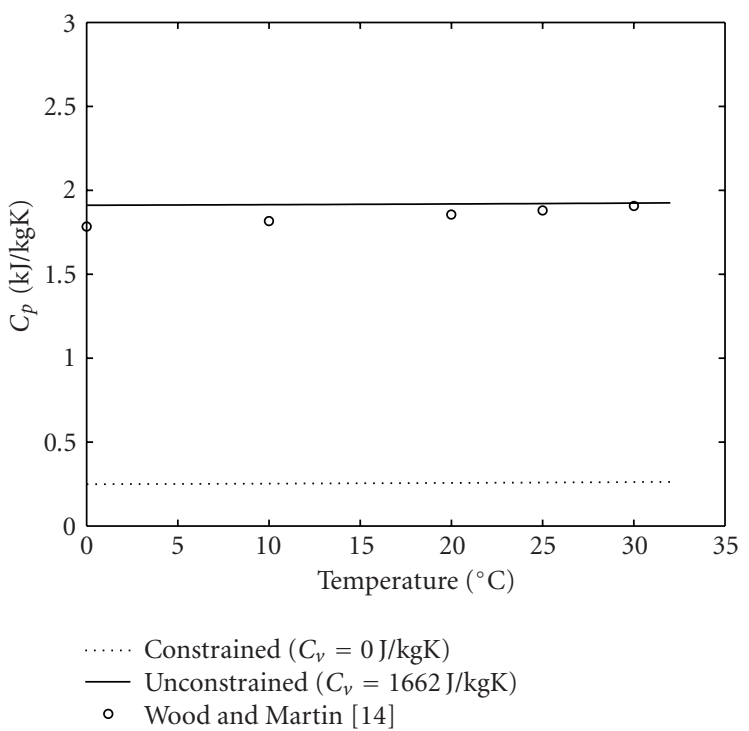

Figure 4.2. Comparison $C_{p}$ for the constrained $\left(C_{v}=0 \mathrm{j} / \mathrm{kgK}\right)$ and unconstrained $\left(C_{v}=1662 \mathrm{~J} / \mathrm{kgK}\right)$ materials. Experimental data is from Wood and Martin [14]. 


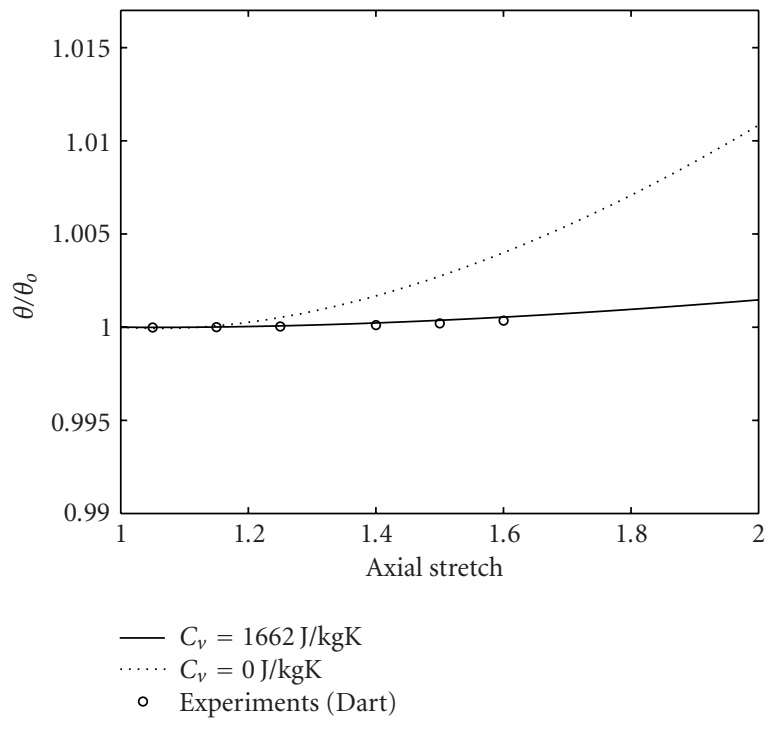

Figure 4.3. The variation of temperature with axial stretch for different $C_{v}$. Experimental data is from Dart (see James and Guth [7]).

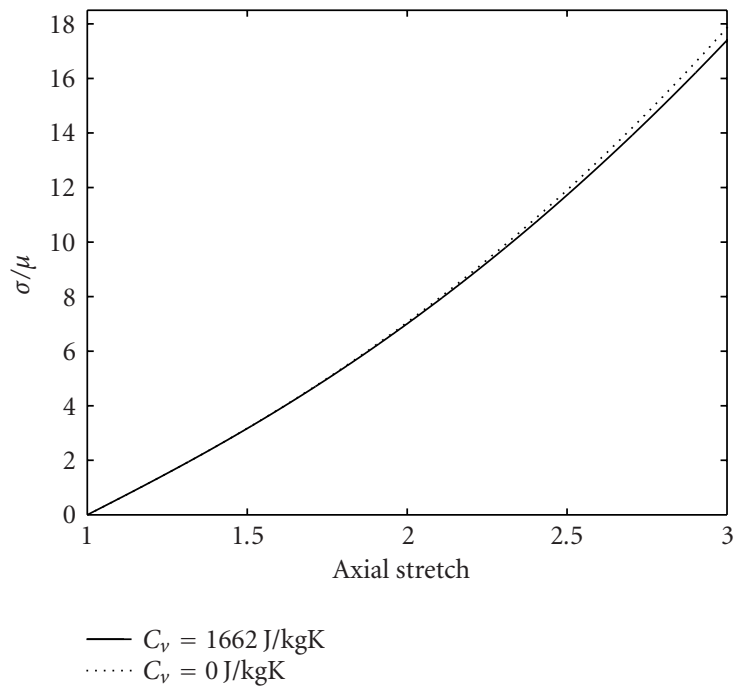

Figure 4.4. The variation of the axial stress $\sigma$ with axial stretch for different $C_{v}$. 


\section{Discussion}

Thus we have shown that it is unnecessary to consider equations of the form $J=\hat{J}(\eta)$ or $J=$ $\hat{J}(\varepsilon)$ as constraints. Indeed, such conditions are easily gone with appropriate Helmholtz potentials of the form

$$
\psi=\hat{\varepsilon}(\mathbf{F})-\theta \hat{\eta}
$$

For peroxide-cured vulcanizate of rubber, we show that a constitutive function of the form (5.1) can be used to model the response. We see that the stress and temperature responses in isothermal and isentropic extension, regardless of the change in $C_{v}$, are almost the same for $\Lambda<1.5$. This implies that, for this value of $\Lambda$, the deformation-entropy constraint or the equivalent form of the Helmholtz free energy can be employed without serious error.

One of the principal motivations for considering constraints is the simplification that it offers for analytical solution of some boundary value problems. However, constraints of the form $J=\hat{J}(\eta)$ and $J=\hat{J}(\varepsilon)$ do not allow for such simplification, and it may be better to simply use an unconstrained thermoelastic model if one is interested in capturing the effects of volume change.

\section{Acknowledgment}

The authors wish to thank the Army Research Office for supporting this research work.

\section{References}

[1] S. Baek and A. R. Srinivasa, Diffusion of a fluid through an elastic solid undergoing large deformation, Internat. J. Non-Linear Mech. 39 (2004), no. 2, 201-218.

[2] H. B. Callen, Thermodynamics and an Introduction to Thermostatistics, 2nd ed., John Wiley \& Sons, New York, 1985.

[3] P. Chadwick, Thermo-mechanics of rubberlike materials, Philos. Trans. Roy. Soc. London Ser. A 276 (1974), no. 1260, 371-403.

[4] P. Chadwick and N. H. Scott, Linear dynamical stability in constrained thermoelasticity. I. Deformation-temperature constraints, Quart. J. Mech. Appl. Math. 45 (1992), no. 4, 641650.

[5] A. E. Green, P. M. Naghdi, and J. A. Trapp, Thermodynamics of a continuum with internal constraints, Internat. J. Engrg. Sci. (1970), no. 8, 891-908.

[6] D. J. Gunton and G. A. Saunders, Stability limits on the Poisson ratio: Application to a martensitic transformation, Proc. Roy. Soc. London Ser. A 343 (1975), 63-83.

[7] H. M. James and E. Guth, Theory of the elastic properties of rubber, Chem. Phys. 11 (1943), no. $10,455-481$.

[8] R. J. Knops and E. W. Wilkes, Theory of elastic stability, Mechanics of Solids (C. Truesdell, ed.), vol. 3, Springer, Berlin, 1984, pp. 125-302.

[9] N. H. Scott, A theorem in thermoelasticity and its application to linear stability, Proc. Roy. Soc. London Ser. A 424 (1989), no. 1866, 143-153.

[10] Linear dynamical stability in constrained thermoelasticity. II. Deformation-entropy constraints, Quart. J. Mech. Appl. Math. 45 (1992), no. 4, 651-662. 
[11] Connections between deformation-temperature and deformation-entropy constraints and near-constraints in thermoelasticity, Internat. J. Engrg. Sci. 34 (1996), no. 15, 16891704.

[12] , Thermoelasticity with thermomechanical constraints, Internat. J. Non-Linear Mech. 36 (2001), no. 3, 549-564.

[13] C. Truesdell and W. Noll, The Nonlinear Field Theories of Mechanics, 2nd ed., Springer-Verlag, Berlin, 1992.

[14] L. A. Wood and G. M. Martin, Compressibility of natural rubber at pressures below $500 \mathrm{~kg} / \mathrm{cm}^{2}$, J. Res. Nat. Bur. Standards 68A (1964), 259-268.

S. Baek: Department of Mechanical Engineering, Texas A\&M University, College Station, TX 77843, USA

E-mail address: baeksi@tamu.edu

A. R. Srinivasa: Department of Mechanical Engineering, Texas A\&M University, College Station, TX 77843, USA

E-mail address: ASrinivasa@mengr.tamu.edu 


\section{Differential Equations \& Nonlinear Mechanics}

\section{An Open Access Journal}

\section{Editor-in-Chief}

K. Vajravelu

USA

Associate Editors

N. Bellomo

Italy

J. L. Bona

USA

J. R. Cannon

USA

S.-N. Chow

USA

B. S. Dandapat

India

E. DiBenedetto

USA

R. Finn

USA

R. L. Fosdick

USA

J. Frehse

Germany

A. Friedman

USA

R. Grimshaw

UK

J. Malek

Czech Republic

J. T. Oden

USA

R. Quintanilla

Spain

K. R. Rajagopal

USA

G. Saccomandi

Italy

Y. Shibata

Japan

Ivar Stakgold

USA

Swaroop Darbha

USA

A. Tani

Japan

S. Turek

Germany

A. Wineman

USA
Website: http://www.hindawi.com/journals/denm/

Aims and Scope

Differential equations play a central role in describing natural phenomena as well as the complex processes that arise from science and technology. Differential Equations \& Nonlinear Mechanics (DENM) will provide a forum for the modeling and analysis of nonlinear phenomena. One of the principal aims of the journal is to promote cross-fertilization between the various subdisciplines of the sciences: physics, chemistry, and biology, as well as various branches of engineering and the medical sciences.

Special efforts will be made to process the papers in a speedy and fair fashion to simultaneously ensure quality and timely publication.

DENM will publish original research papers that are devoted to modeling, analysis, and computational techniques. In addition to original full-length papers, DENM will also publish authoritative and informative review articles devoted to various aspects of ordinary and partial differential equations and their applications to sciences, engineering, and medicine.

\section{Open Access Support}

The Open Access movement is a relatively recent development in academic publishing. It proposes a new business model for academic publishing that enables immediate, worldwide, barrier-free, open access to the full text of research articles for the best interests of the scientific community. All interested readers can read, download, and/or print any Open Access articles without requiring a subscription to the journal in which these articles are published.

In this Open Access model, the publication cost should be covered by the author's institution or research funds. These Open Access charges replace subscription charges and allow the publishers to give the published material away for free to all interested online visitors.

\section{Instructions for Authors}

Original articles are invited and should be submitted through the DENM manuscript tracking system at http://www.mstracking.com/ denm/. Only pdf files are accepted. If, for some reason, submission through the manuscript tracking system is not possible, you can contact denm.support@hindawi.com.

Hindawi Publishing Corporation

410 Park Avenue, 15th Floor, \#287 pmb, New York, NY 10022, USA
HINDAWI 


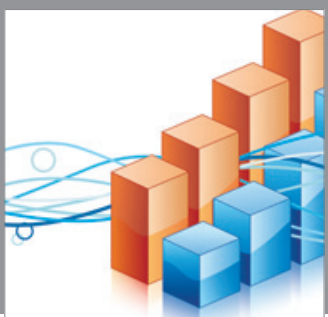

Advances in

Operations Research

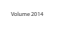

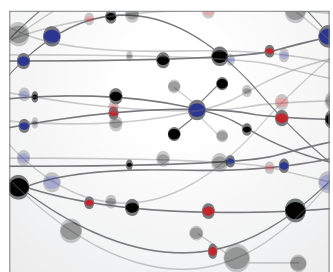

\section{The Scientific} World Journal
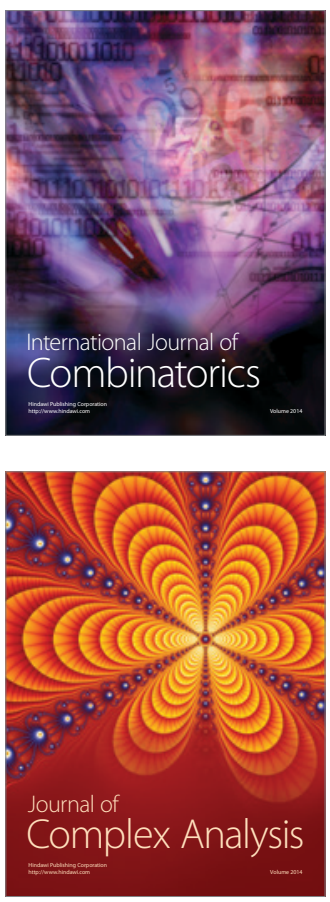

International Journal of

Mathematics and

Mathematical

Sciences
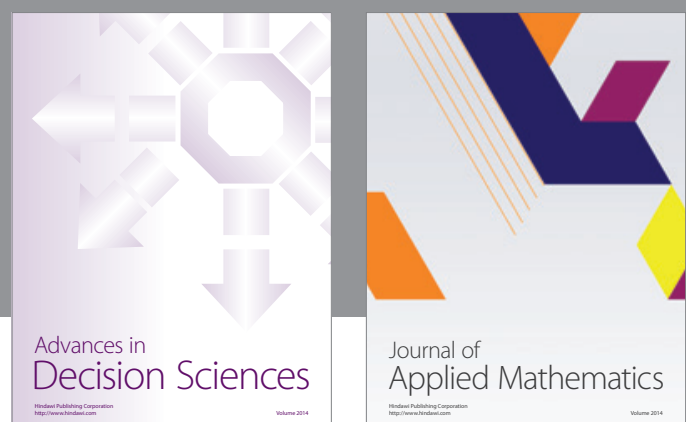

Journal of

Applied Mathematics
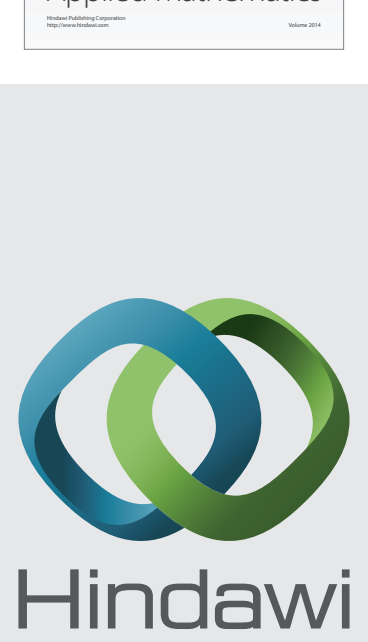

Submit your manuscripts at http://www.hindawi.com
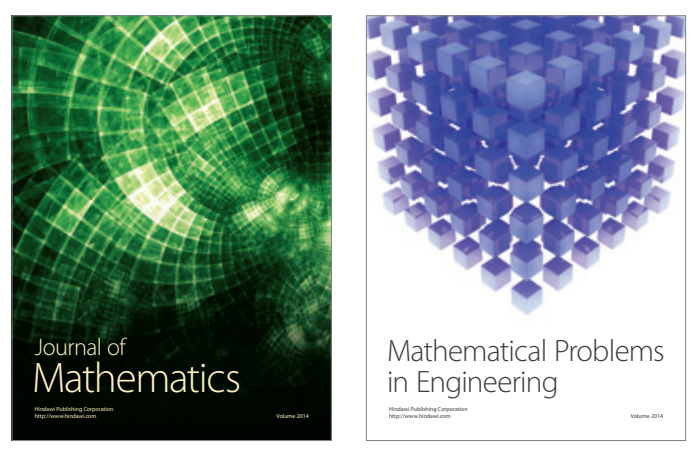

Mathematical Problems in Engineering
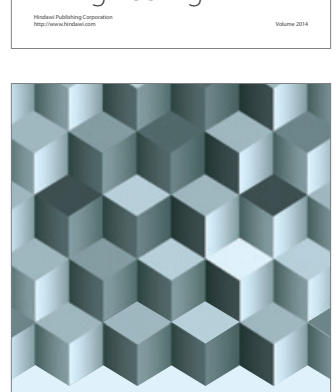

Journal of

Function Spaces
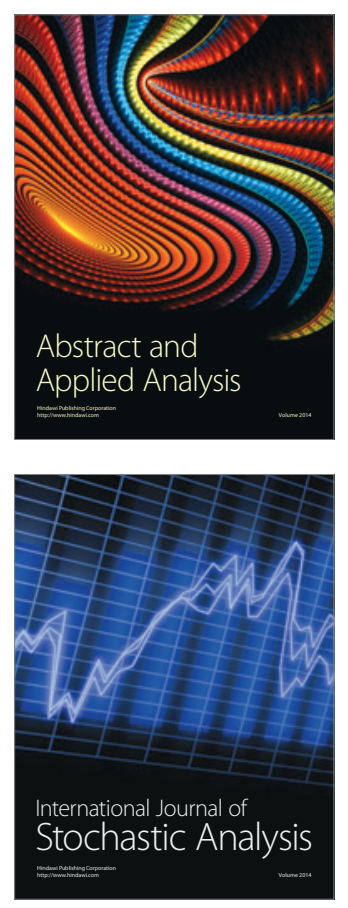

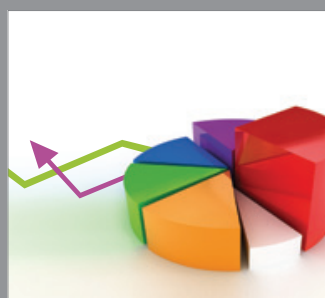

ournal of

Probability and Statistics

Promensencen
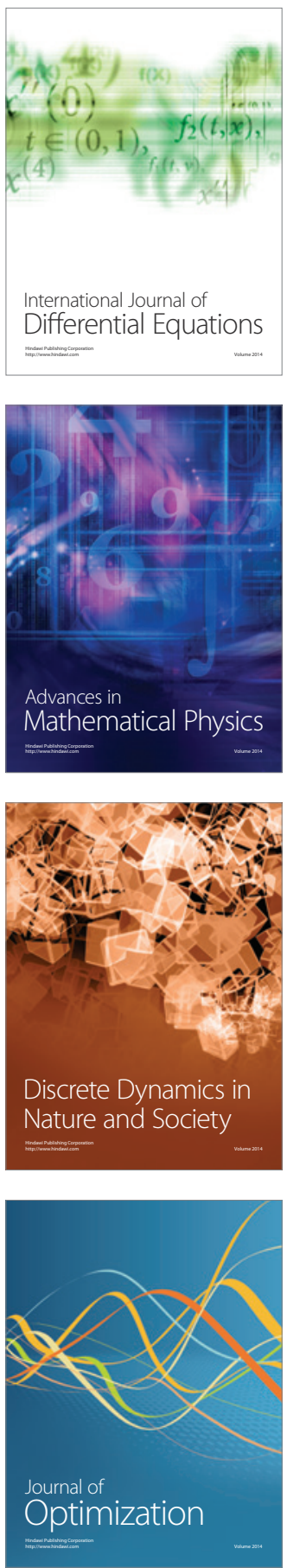